\title{
Effects of Inter-Particle Frictional Coefficients on Evolution of Contact Networks in Landslide Process
}

\author{
Lian Jiang1, Enlong Liu ${ }^{1,2 *}$, Jianqiu Tian ${ }^{1}$, Xiaoqiong Jiang ${ }^{1}$ \\ ${ }^{1}$ State Key Laboratory of Hydraulics and Natural River Engineering, College of Water Resource and Hydropower, Sichuan \\ University, Chengdu, China \\ ${ }^{2}$ Northwest Institute of Eco-Environment and Resources, State Key Laboratory of Frozen Soil Engineering, CAS, Lanzhou, China \\ Email: *liuenlong@lzb.ac.cn
}

How to cite this paper: Jiang, L., Liu, E.L., Tian, J.Q. and Jiang, X.Q. (2017) Effects of Inter-Particle Frictional Coefficients on Evolution of Contact Networks in Landslide Process. Engineering, 9, 917-936. https://doi.org/10.4236/eng.2017.911055

Received: October 11, 2017

Accepted: November 24, 2017

Published: November 27, 2017

Copyright $\odot 2017$ by authors and Scientific Research Publishing Inc. This work is licensed under the Creative Commons Attribution International License (CC BY 4.0).

http://creativecommons.org/licenses/by/4.0/

\begin{abstract}
During the process of landslide, its dynamic mechanism is important to understand and predict these kinds of natural hazard. In this paper, a new method, based on concepts of complex networks, has been proposed to investigate the evolution of contact networks in mesoscale during the sliding process of slope. A slope model was established using the discrete element method (DEM), and influences of inter-particle frictional coefficients with four different values on dynamic landslides were studied. Both macroscopic analysis on slope landslide and mesoanalysis on structure evolution of contact networks, including the average degree, clustering coefficient and $\mathrm{N}$-cycle, were done during the process of landslide. The analysis results demonstrate that: 1 ) with increasing inter-particle frictional coefficients, the displacement of slope decreases and the stable angle of slope post-failure increases, which is smaller than the peak internal frictional angle; 2) the average degree decreases with the increase of inter-particle frictional coefficient. When the displacement at the toe of the slope is smaller, the average degree there changes more greatly with increasing inter-particle frictional coefficient; 3 ) during the initial stage of landslide, the clustering coefficient reduces sharply, which may leads to easily slide of slope. As the landslide going on, however, the clustering coefficient increases denoting increasing stability with increasing inter-particle frictional coefficients. When the inter-particle frictional coefficient is smaller than 0.3 , its variation can affect the clustering coefficient and stable inclination of slope post-failure greatly; and 4) the number of 3-cycle increases, but 4-cycle and 5-cycle decrease with increasing inter-particle frictional coefficients.
\end{abstract}

\section{Keywords}

Evolution of Contact Network, Landslide, Complex Network, Inter-Particle 


\section{Introduction}

Slope failure is a common geological hazard, which is a changing progress with time and space, including initial stage, landslide stage and stable stage post failure [1]. Numerical methods have been widely used to predict the slope stability, study failure patterns and dynamic rules, including limit equilibrium method [2] [3], finite element method [4] and discrete element method [5]. The finite element method based on continuum mechanics is hardly employed to simulate the process of landslide, and although the discrete element method can be used to study the macroscopic rules of landslide, such as movement velocity and slide distance, it can't provide the bridging relationships between the contacting relationships between sliding blocks and their evolution rules in mesoscale and macroscopic movement rules during the process of landslide. Therefore, studies on mesostructures and evolution of contact networks are necessary to be done to provide a new method to analyze the macroscopic mechanism of landslide.

Discrete element method is a numerical method based on non-continuous medium analysis, which has been widely used in analysis of geotechnical problems [6] [7] [8] [9] [10]. Through defining the meso parameters of particles, it can be used to not only solve macroscopic problems, but also save much information about soil particles. Wang et al. [11] found that joint connectivity has great influences on stability of rockmass slope by defining different values of joint connectivity. Scholtès and Donze [12] studied the effects of joints with different angles of inclination on slope failure through acquiring particle velocity in the process of landslide. Chen and Liu [13] compared the numerical simulations with experimental results to study the properties of failure, and found that the angle at failure obtained by simulation is the same as that obtained by tests. Although DEM has been used to simulate the sliding process of landslide, macroscopic process of landslide should be analyzed physically in detail from the meso scale of soil grains and meso structure network, which will be explored primarily here in the viewpoint of complex network method.

Complex network is a method used to study the physical process or data, which is widely used in economics [14], sociology [15], medical science [16] and physics [17]. Recently, complex network has also been gradually employed to analyze the physical and mechanical properties of granular matter. Peters et al. [18] divided contact network into strong network and weak network according to the values of contact force among particles, and quantified the length and amounts of force chains. Walker and Tordesillas [19] investigated the deformational features of dense materials upon loading from the perspective of complex network, and studied the development of network structures and loss of connectivity in the process of compression. Tordesillas et al. [20] performed biaxial compressional tests 
on three different samples and analyzed the evolution of network structure and contact force, which demonstrated that the resolve of connectivity and structure of three sub-networks leads to failure of samples. Papadopoulos et al. [21] used the multi-layer network as framework to quantify the meso parameters of samples under compression.

Although many studies have been done on dynamics and evolving rules of landslides, attentions are usually paid on macroscopic description of landslide. Methods based on complex network have been used to analyze compression process of dense samples, but few studies have been reported on contact network analysis of landslide process in a perspective of complex network. In view of this, discrete element method (DEM) is employed here to construct slope model for computational analysis, based on which the influences of inter-particle frictional coefficients on process of landslide is investigated. In addition, from the perspective of complex network, the average degree, clustering coefficient and N-cycle are also explored in detail during the process of landslide.

\section{DEM Model of Slope}

\subsection{Establishment of Slope Model}

In the paper, PFC (particle flow code), one kind of DEM, is used to perform the sliding analysis of slope because it can simulate the landslide process and track the particles' state easily. For PFC in 2D, the particles are assumed to be rigid circles and the deformation can occur at the contacting points among these particles. The particles obey Newton' law of motion, and the computational principle can be found in references [5]. The contact-bonded model was used in the simulation, with particle sizes ranging from $3 \mathrm{~mm}$ to $6 \mathrm{~mm}$, both normal stiffness and tangential stiffness are $50 \mathrm{MPa}$, contact bond strength at initial state is $50 \mathrm{MN} / \mathrm{m}$, inter-particle frictional coefficient $(f)$ at initial state is 0.9 , and initial void ratio is 0.16 . Similar to centrifuge model, an amplified gravity field is used in this simulation [22]. Under $200 \mathrm{~g}$ of gravitational field, a rectangular coordinate system, with positive $\mathrm{x}$ rightwards and positive $\mathrm{y}$ upwards, has been established and a rectangular model with $1.8 \mathrm{~m}$ in length and $0.6 \mathrm{~m}$ in height was generated. By deleting the redundant particles, a slope model with 0.8 of ratio of slope can be established. The generated slope has free upper surface, frictionless surfaces of lateral walls and bottom surface. In order to observe and track the displacement of soil particles, the particles were grouped as small square zones with $0.5 \mathrm{~m}$ in length of one side, each including about 30 particles, as shown in Figure 1.

According to Mohr-Coulomb criterion, the factors causing slope failure include reduction of internal frictional angles [23], loss of cohesive forces [24] and increase of pore water pressure [25]. In the following, the influences of inter-particle frictional coefficients on dynamic process of landslide of unbounded soil slope will be studied with variations of inter-particle frictional coefficients of $0.9,0.5,0.3$ 


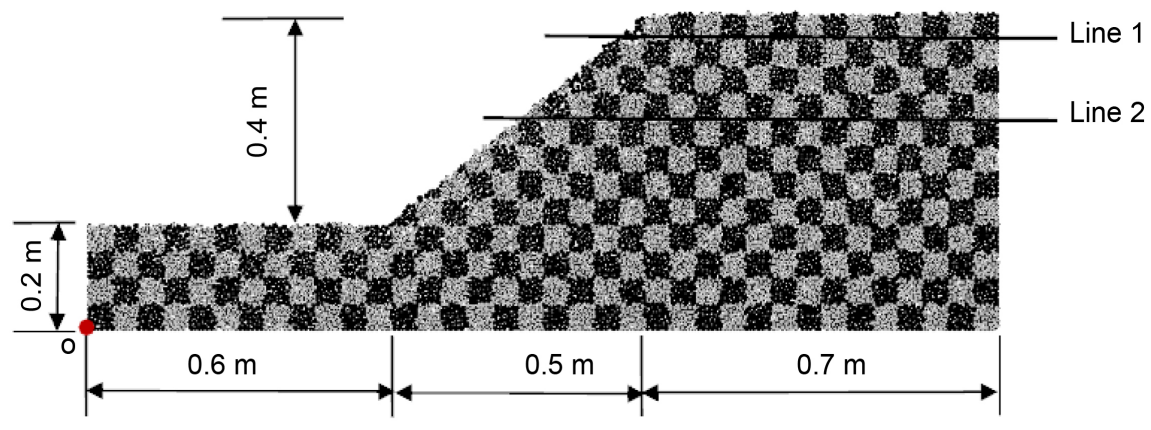

Figure 1. Slope model.

and 0.2 when the contact bond strength between soil particles is zero.

\subsection{Calibration of Parameters of Biaxial Tests}

Here studies are focused on variations of inter-particles frictional coefficients affecting dynamic process of landslide when bond strength between soil particles quickly reduces to be zero. Therefore, it is the first step to measure the mechanical behaviors of unbounded soils with different inter-particle frictional coefficients for biaxial compression tests. The generated sample is $0.8 \mathrm{~m}$ in height and $0.4 \mathrm{~m}$ in length including 4000 particles, and the contact bond strength between soil particles is zero, the initial void ratio is 0.16 . The inter-particle frictional coefficients are $0.9,0.5,0.3$, and 0.2 , respectively, and lateral confining pressures employed are $50 \mathrm{kPa}, 100 \mathrm{kPa}, 200 \mathrm{kPa}$, and $400 \mathrm{kPa}$, respectively, and when the axial compressional strain reaches $20 \%$ with loading rate of $1 \% / \mathrm{min}$, it stops. Figure 2 and Figure 3 present the deviatoric stress-axial strain curves and volumetric strain-axial strain curves of different inter-particle frictional coefficients at the same lateral confining pressures respectively, and Figure 4 presents the relationship between internal frictional angles in peak values and inter-particle frictional coefficients. From Figure 2 and Figure 3, we can find that with increasing of inter-particle frictional coefficients, the peak deviator stress also increase with more heavily strain softening, and the samples dilate more heavily; and dilatancy becomes greater with increasing inter-particle frictional coefficient under the same lateral confining pressures. The reason for this phenomena is that: the samples are relative dense at the beginning of applying deviatoric stress, and only particles displace, rearrange and dilate leading to bear the external load; the particles with higher inter-particle frictional coefficient can bear more external loading between their contacting points, followed by stress decrease accompanied by dilatancy; with increasing axial strain, the samples contract within small axial stress and dilate until failure, which result loose zone along a band in the sample and slip along this band, thus the inter-particle frictional coefficient hardly affects the residual strength and samples with higher inter-particle frictional coefficients have bigger stress reduction. From Figure 4, we know that the internal frictional angles at peak deviator stress increase and the shear strength 


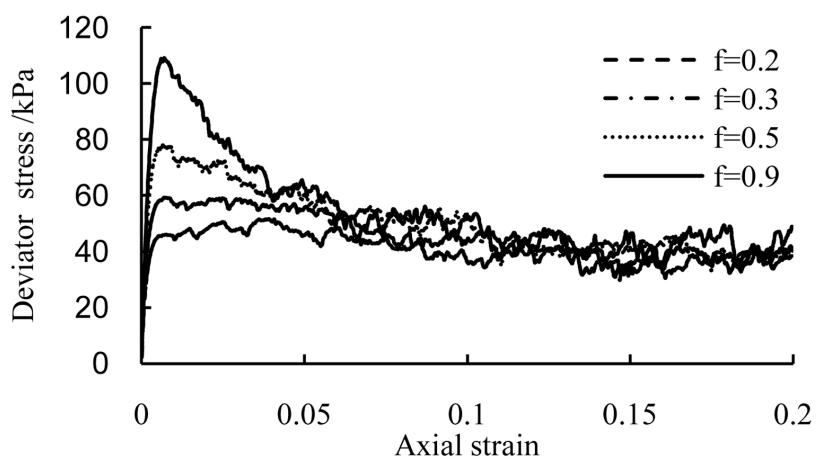

(a)

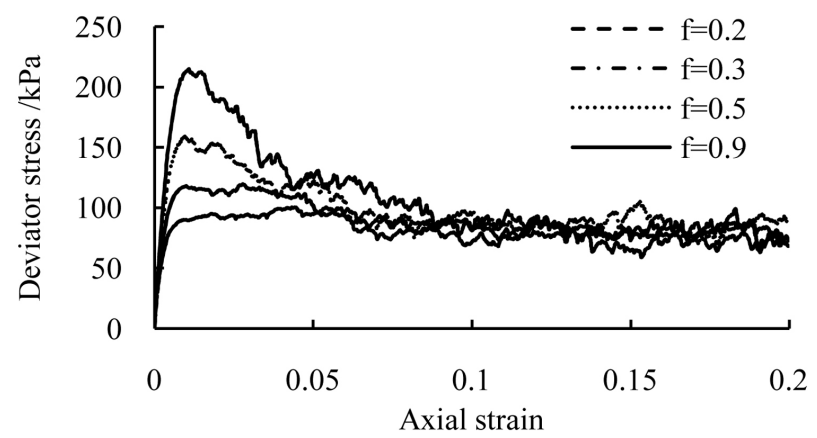

(b)

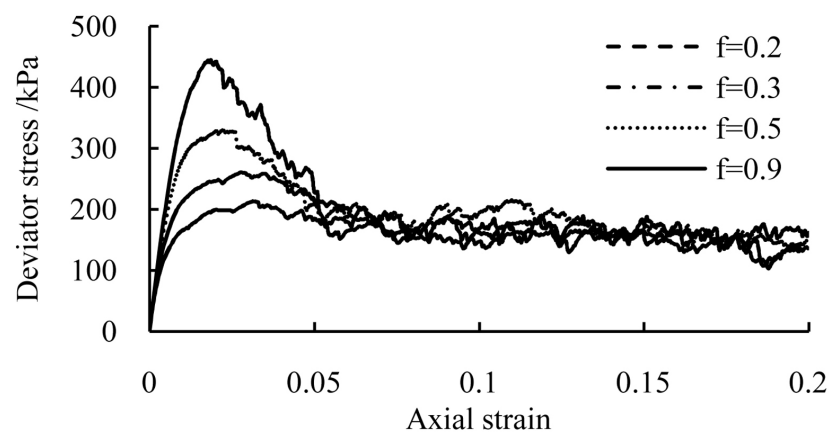

(c)

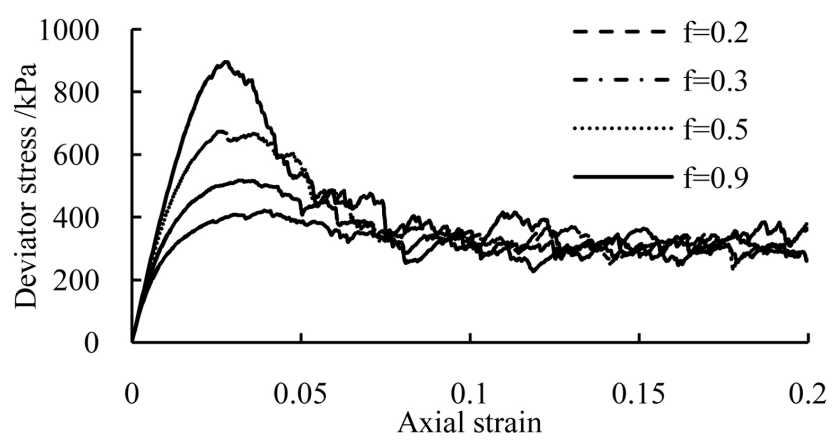

(d)

Figure 2. The deviatoric stress-axial strain curves of different inter-particle frictional coefficients at the same lateral confining pressure. (a) Lateral confining pressure of $50 \mathrm{kPa}$; (b) Lateral confining pressure of $100 \mathrm{kPa}$; (c) Lateral confining pressure of $200 \mathrm{kPa}$; (d) Lateral confining pressure of $400 \mathrm{kPa}$. 


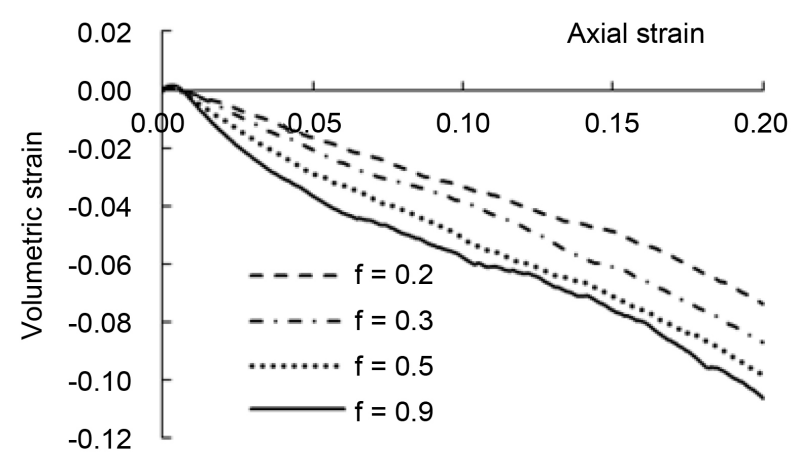

(a)

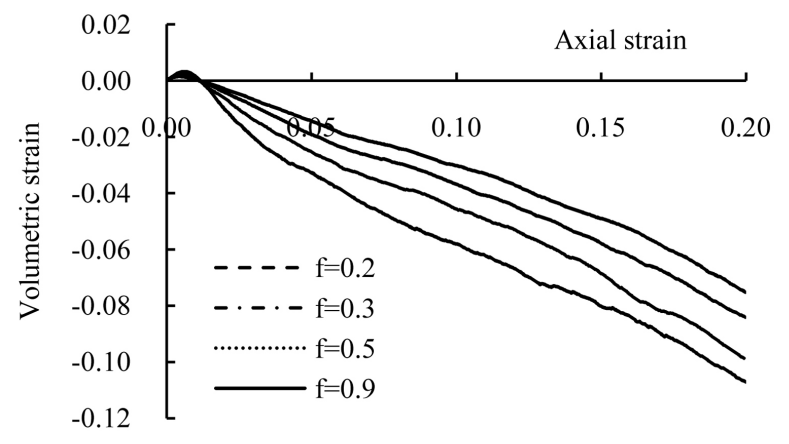

(b)

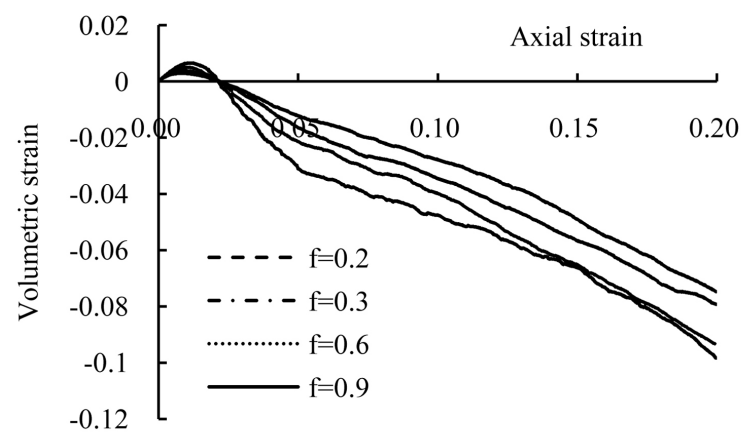

(c)

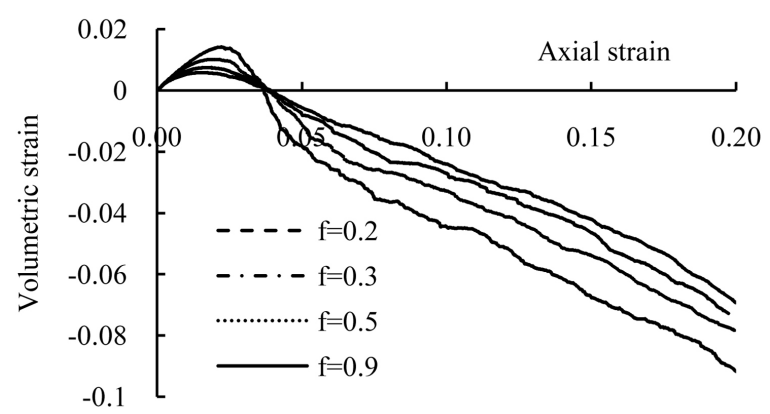

(d)

Figure 3. The volumetric strain-axial strain curves of different inter-particle frictional coefficients at the same lateral confining pressure. (a) Lateral confining pressure of $50 \mathrm{kPa}$; (b) Lateral confining pressure of $100 \mathrm{kPa}$; (c) Lateral confining pressure of $200 \mathrm{kPa}$; (d) Lateral confining pressure of $400 \mathrm{kPa}$. 


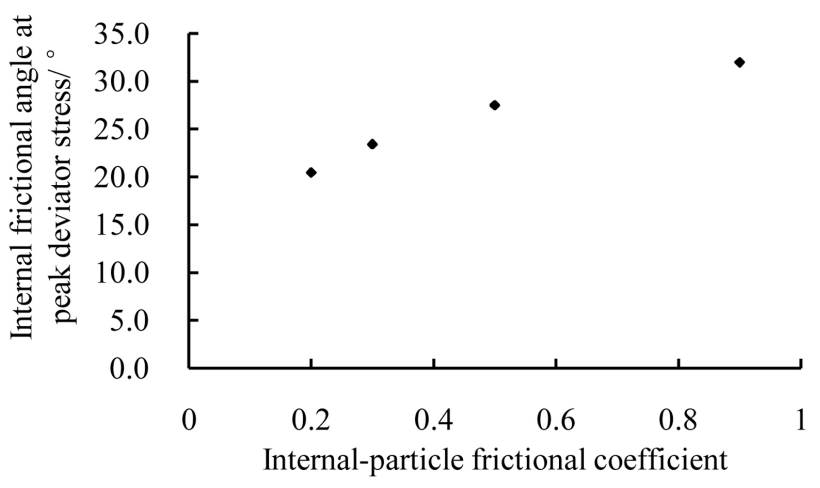

Figure 4. The relationship between internal frictional angles in peak values and inter-particle frictional coefficients.

of the samples also increase with increasing inter-particle frictional coefficient.

\section{Instability Analysis of Slope}

\subsection{Displacement Analysis}

As shown in Figure 1, Line 1 is located in the top of slope and Line 2 is located in the middle of slope. Take Point $\mathrm{O}$ as origin of coordinate as shown in Figure 1 , we can trace the displacement paths on the particles located on the grid nodes, as shown in Figure 5. From Figure 5, we know that the particles of grid nodes close to slope surface have bigger displacements, and after failure, the particles displace to new positions lower than the initial ones. When the inter-particle frictional coefficient, denoted by $f$, is 0.2 , the particles of grid nodes located within $\mathrm{x}<$ $1.45 \mathrm{~m}$ displace downward along the slope surface; when $f$ is 0.3 , the particles of grid nodes located within $\mathrm{x}<1.35 \mathrm{~m}$ displace downward along the slope surface; when $f$ is 0.6 , the particles of grid nodes located within $\mathrm{x}<1.25 \mathrm{~m}$ displace downward along the slope surface; and when $f$ is 0.9 , the particles of grid nodes located within $\mathrm{x}<1.20 \mathrm{~m}$ displace downward along the slope surface. The same phenomena appear for particles located on Line 2. When the values of $f$ are different, the number of particles located the small grids slide downwards along the slope surface decreases and the displacement of slope slide also decreases with $f$ increasing.

\subsection{Analysis on Geometrical Shape of Slope Post-Failure at a New Stable State}

After the slopes fail, the particles slide and adjust their position timely and arrive at their new positions, and thus the geometrical shape of the slope at the new stable state can be formed as shown in Figure 6. From Figure 6, we know that the slope surface of slope at stable state after failure does not go through the toe of initial slope, but about the middle of surface of initial slope. When $f$ is 0.2 , the stable angle of slope post-failure is $18.4^{\circ}$, which is slightly smaller than its internal frictional angle $20.5^{\circ}$ at peak deviatoric stress. These phenomena appear for other values of $f 0.3,0.5$ and 0.9 . When $f$ is different, slope slides more greatly 


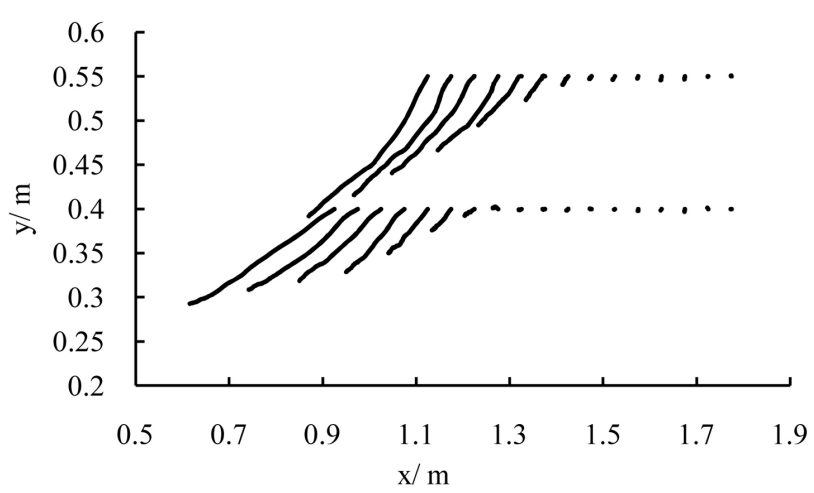

(a)

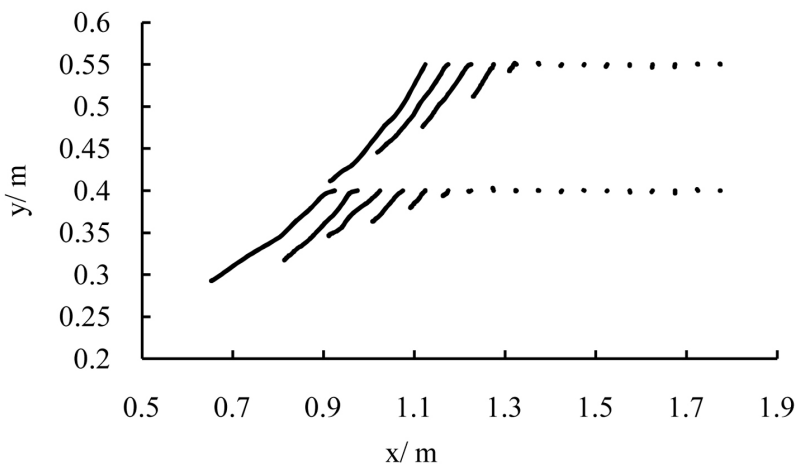

(b)

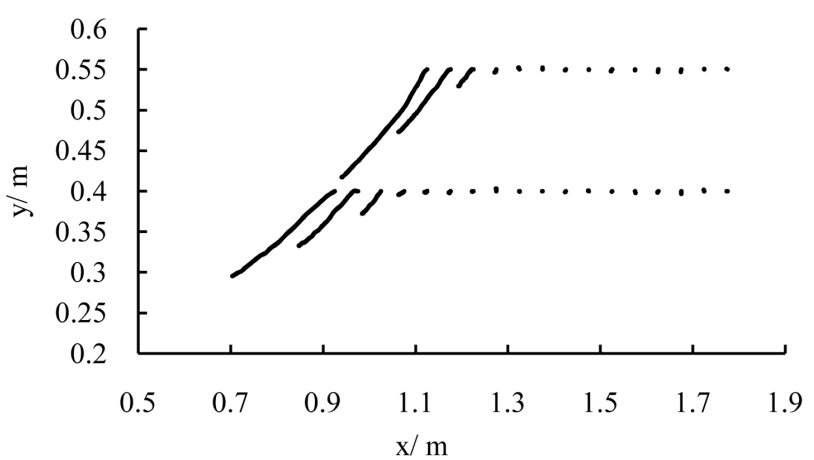

(c)

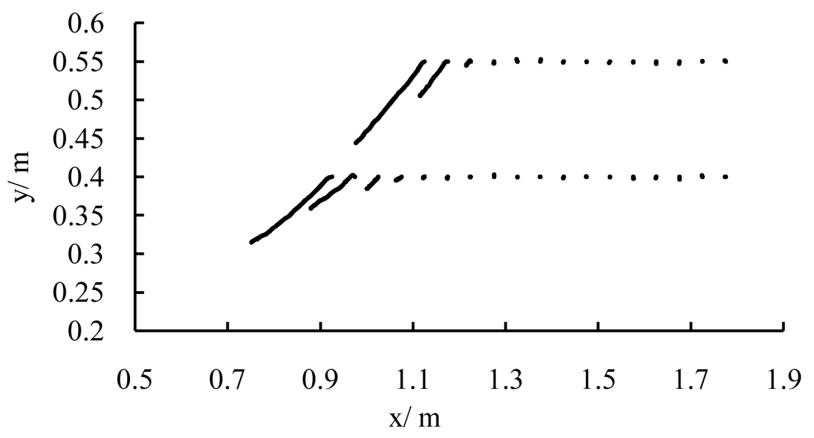

(d)

Figure 5. Displacement paths of particles on Line 1 and Line 2 ( $f$ denoting inter-particle frictional coefficients). (a) $f=0.2$; (b) $f=$ 0.3 ; (c) $f=0.5$; (d) $f=0.9$. 


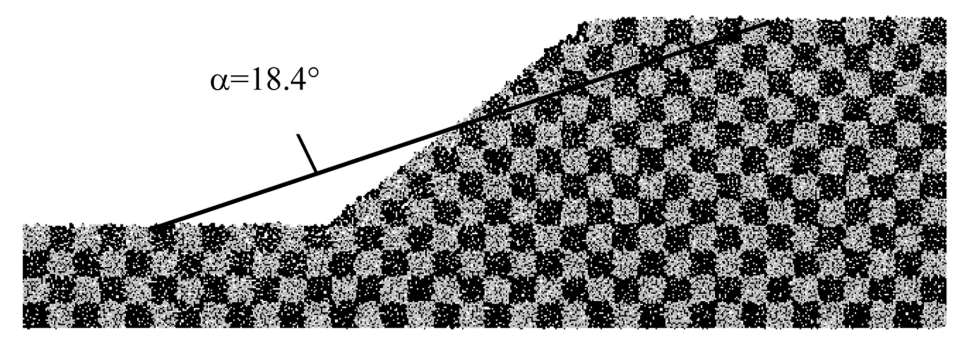

(a)

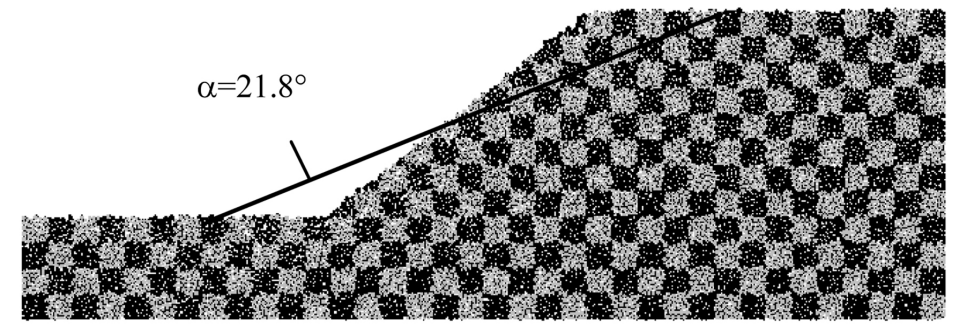

(b)

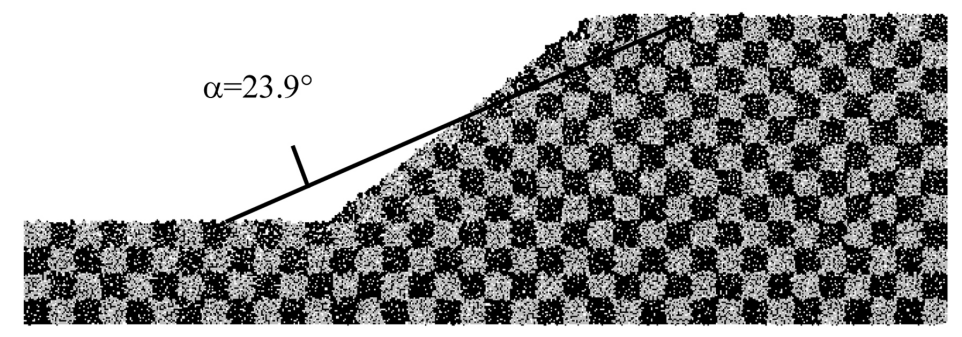

(c)

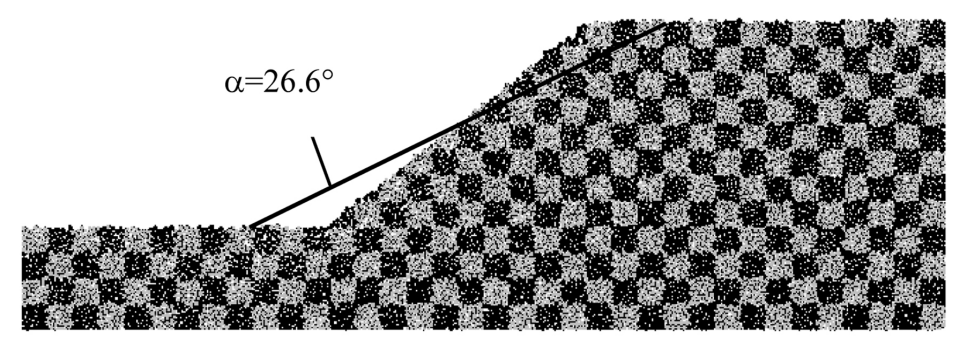

(d)

Figure 6. Geometrical shape of slope post-failure at stable state. (a) $f=$ 0.2 ; (b) $f=0.3$; (c) $f=0.5$; (d) $f=0.9$.

and the volume of landslide is huger with decreasing $f$, which leads to smaller angle of slope formed at stable state after failure.

\subsection{Displacement at Slope Toe}

The deformation of slope during landslide is very important for dynamic analysis, which will be analyzed here at slope toe here, as shown in Figure 7. From Figure 7, we know that with increasing $f$, the displacement at slope toe reduces. When $f$ is 0.9 , the initial displacement there is smaller meaning that the slope 


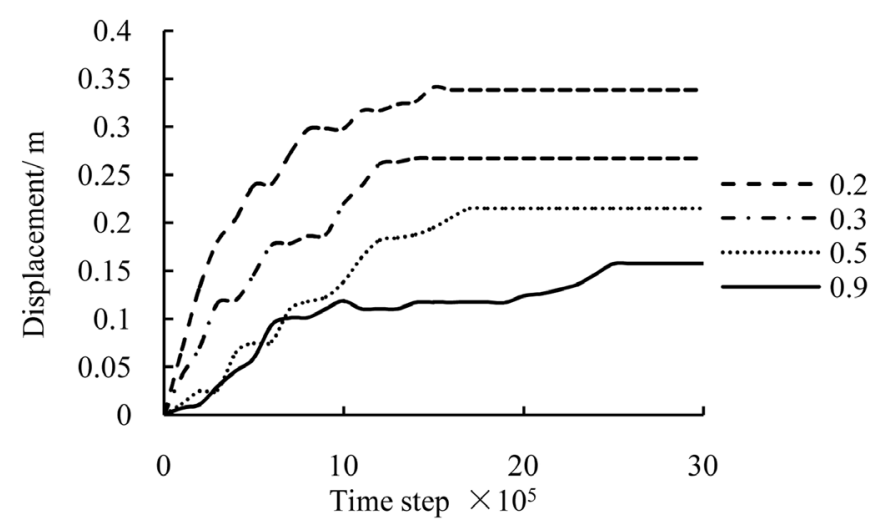

Figure 7. The displacement of slope toe.

slides gradually, followed by quick movement and the displacement there increases soon, and then the speed of movement becomes smaller tending to a new stable state. When $f$ is $0.5,0.3$ and 0.2 , respectively, however, the displacement at slope toe firstly increases quickly meaning the slope toe slides sharply and then its speed arrive at a new stable value. Therefore, when $f$ is 0.9 , the movement at slope toe can be divided into three stages including slow movement, quickly increasing rate of movement and reducing movement rate to a stable value; but, when $f$ is $0.5,0.3$ and 0.2 , respectively, the movement at slope toe can be divided into two stages including quick movement and the decreasing speed tending to a new stable value. The reason for this is maybe that: at $f=0.9$, at the initial stage, particles near slope crest will first slide and drive the soil particles below them to slide gradually, and this process needs some time for the particles adjusting their stress state to balance external loads transferred from adjacent particles, which leads to small amounts of particles slide towards slope toe due to their bigger internal frictional angles at peak deviatoric stress and higher shear strength; with increasing computational steps, more particles slide, which cause more particles quickly slide to slope toe and thus form a new stable state.

\section{Evolution Analysis of Contact Network in the Process of Landslide}

In the contact network, a vertex denotes a single particle, and an edge linking the center of two particles denotes there is a contact between them. When landslide occurs, the particles will rearrange and adjust their positions, which can reflect the evolution of contact networks of these particles, such as loss of old contacts and formation of new ones. Recurring to some concepts from complex network, including the average degree of contact network, clustering coefficient and N-cycle, we can investigate the mesostructures varying with the process of landslide, which can help in understanding the dynamic mechanism of landslide.

\subsection{The Evolution of Average Degree}

The degree of a vertex [26] is the basic characteristic of network, which means 
the number of edges associated with the vertices and has the similar meaning as the coordinate number for granular matter. The value of degree among the particles is relatively high for the small amounts of particles, which is called high degree vertices of particles. The average degree, also called coordinate number, represents the average value of degree of all the particles in the network, which can be linked with the number of particles as follows,

$$
k=\frac{1}{N} \sum_{i, j=1}^{N} a_{i j}
$$

in which $N$ is the number of particles, $a_{i j}$ is adjacent matrix, which means that $a_{i j}=$ 1 when particles $i$ and $j$ contact, otherwise $a_{i j}=0$.

The evolution of average degree with time steps at different values of $f$ is shown in Figure 8. From Figure 8, we know that at initial stage of landslide, the average degrees at different values of $f$ decrease quickly, the reason for that is the reduction of number of contacting particles and the network density; and with the increase of time steps, particles rearrange, which makes the curves of average degrees slightly fluctuate, and when the new stable state is reached, the average degree will not change. At initial stage, when $f$ is equal to $0.9,0.5,0.3$ and 0.2 , the average degree $\mathrm{k}$ is $4.231,4.234,4.238$ and 4.242 respectively. Therefore, at initial stage, with increasing values of $f$, the average degree decreases and network density also decreases; when the new stable state is reached, both average degree and network density decrease with increasing values of $f$.

\subsection{The Evolution of Clustering Coefficient}

The clustering coefficient, also called transitivity [27] is a typical property of network, which describes the probability of adjacent vertices for the points in network. Three vertices adjacent each other form a triangle, which can resist rotation among them, so the clustering coefficient can be used to predict the stability of complex structure. The clustering coefficient of a vertex (or a particle in granular matter) is defined as the ratio of the number of triangles embodying it to that of a connected triple centering it, in which a triangle is a set of three vertices with edges between each pair of vertices and a connected triple is a set of

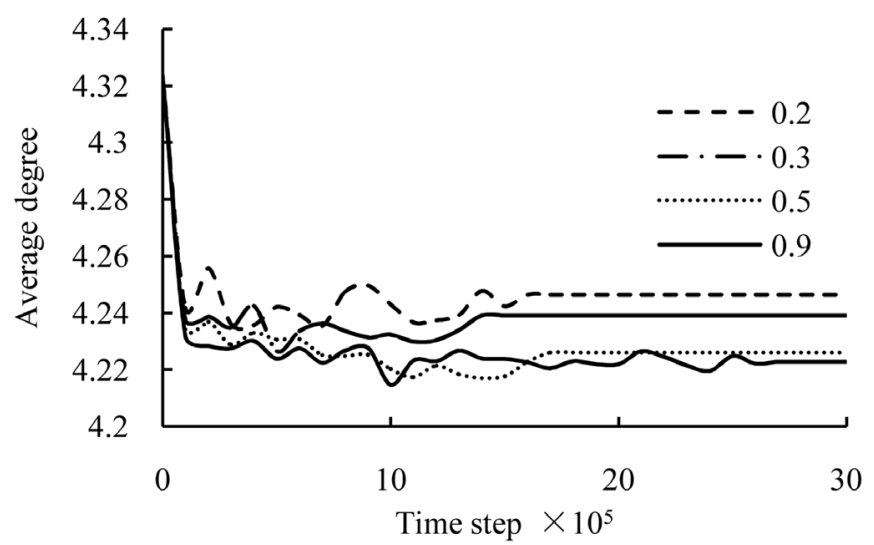

Figure 8. Evolution of average degree. 
three vertices where each vertex can be reached from each other (directly or indirectly) as shown in Figure 9, and can be expressed as follows,

$$
C(i)=\frac{\sum_{k>j} a_{i j} a_{i k} a_{j k}}{\sum_{k>j} a_{i j} a_{i k}}
$$

A vertex in network has a greater coefficient of cluster meaning that there are more triangles embodying and the structure composing this vertex is more stable. The clustering coefficient of a network is defined as the average value of clustering coefficient of the vertex in the network, which can be used to denote the whole stability of the network. The evolving rules of clustering coefficient of the network with computational time steps are shown in Figure 10 in the process of landslide.

From Figure 10, we can find that at initial stage, the clustering coefficient for the different values of $f$ decreases quickly, which means the stability of network also decreases and the slope easily fails; with landslide going on, their values fluctuate slightly; and when the soil particles reach a new stable position, the clustering coefficient will not change with increasing time steps. With increasing values of $f$, the computational time steps needed to reach the stable state will also increase, and the values of clustering coefficient at stable state increases, which means the stability of slope also increases.

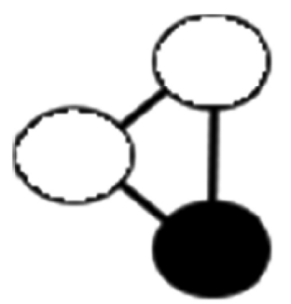

(a)

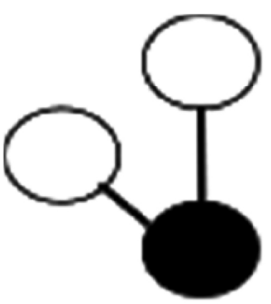

(b)

Figure 9. A triangle and a connected triple. (a) A triangle; (b) A connected triple.

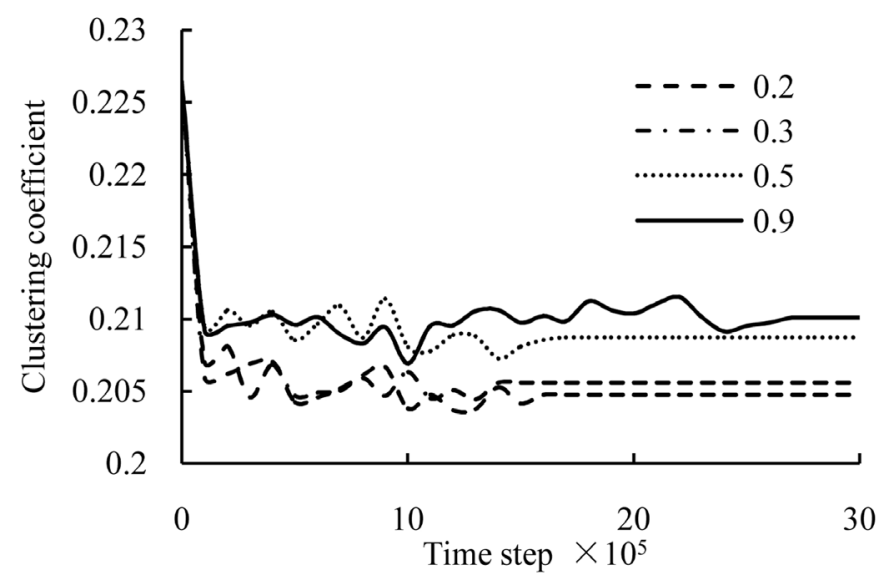

Figure 10. Evolution of clustering coefficient. 


\subsection{N-Cycle}

$\mathrm{N}$-cycle is the shortest closed path with $\mathrm{N}$ in length in an undirected network [28]. In network formed in granular matter, the particles contact each other and form many structures with closed cycles, which sustain together the stability of network. For the three types of structures cycles shown in Figure 11, 3-cycle has the most stability, 4-cycle has the most unstability, and 5-cycle force in between, and the reason for this is that compared with the force cycles having odd number $\mathrm{N}$, the force cycles with even number $\mathrm{N}$ rotate more easily [20]. The failure process of slope is the rearrange process of particles, which will result the loss of old contacts and formation of new contacts, and both structure and number of cycles will change timely.

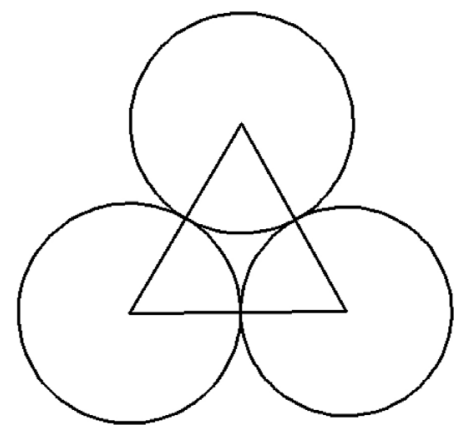

(a)

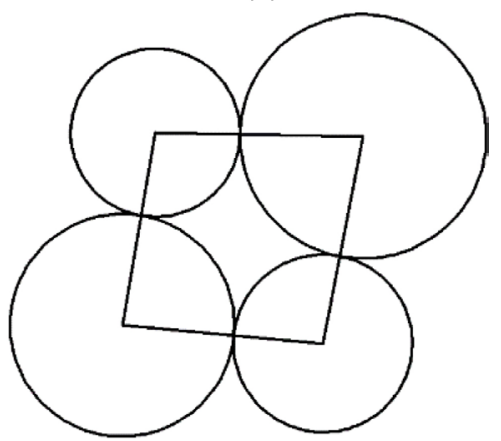

(b)

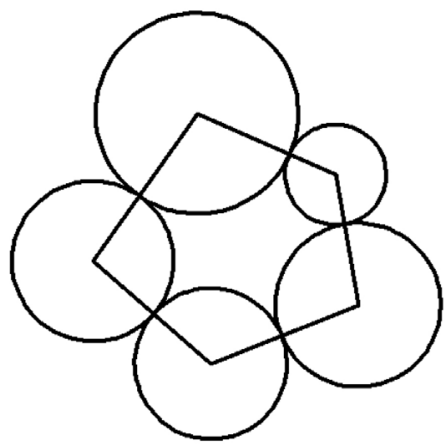

(c)

Figure 11. Structures of N-cycles. (a) 3-cycle; (b) 4-cycle; (c) 5-cycle. 
At different values of $f$, the evolution of 3-cycle, 4-cycle and 5-cycle with computational time steps are shown in Figure 12. From Figure 12, we can find that the 3-cycle has the greatest number and 5-cycle has the least one. At initial stage of landslide, the number of 3-cycle decreases, but the numbers of 5-cycle and 4-cycle (except for $f=0.9$ ) also increase. With computational time steps increase, however, the numbers of 3-cycle, 4-cycle and 5-cycle tend to be stable. The reason for this is that: before initiating landslide, the network structure of soil particles of slope is shown as Figure 13(a), where the number of 3-cycle is most and the slope has the most stability; during the process of landslide, the network

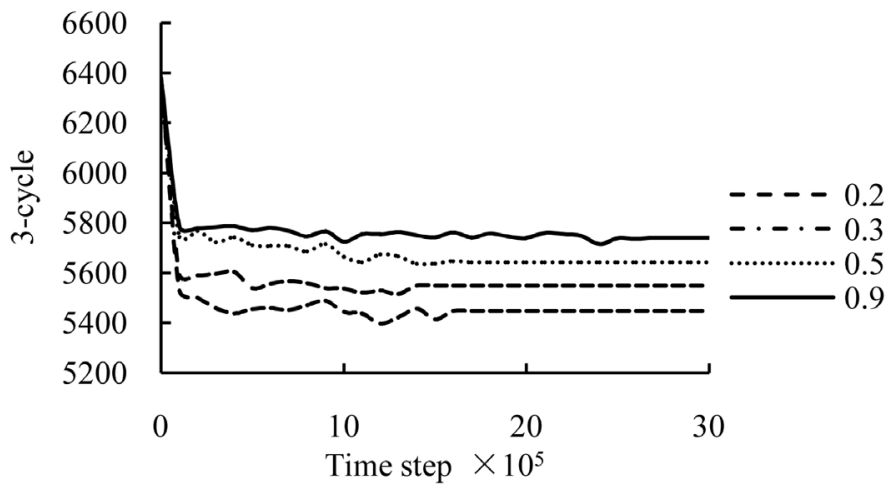

(a)

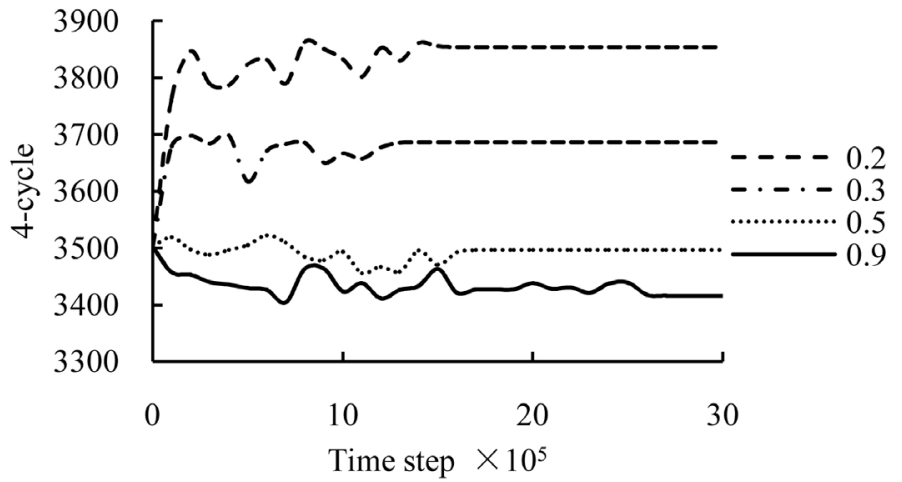

(b)

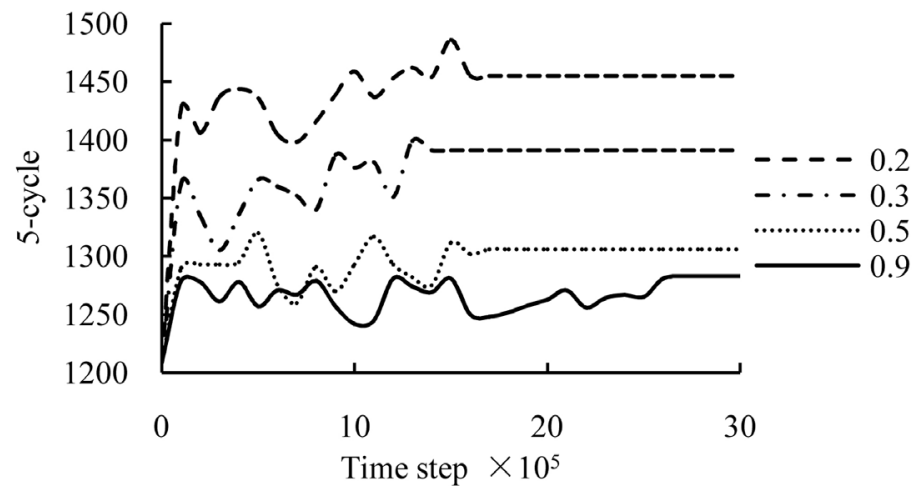

(c)

Figure 12. Evolution of $\mathrm{N}$-cycle with computational time steps. (a) Evolution of 3-cycle; (b) Evolution of 4-cycle; (c) Evolution of 5-cycle. 


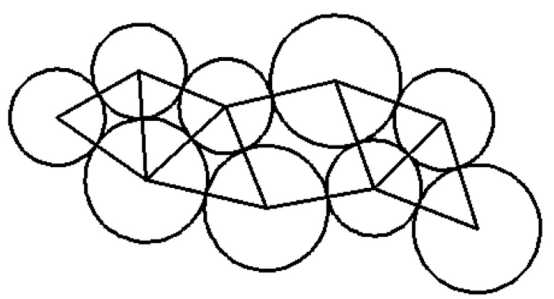

(a)

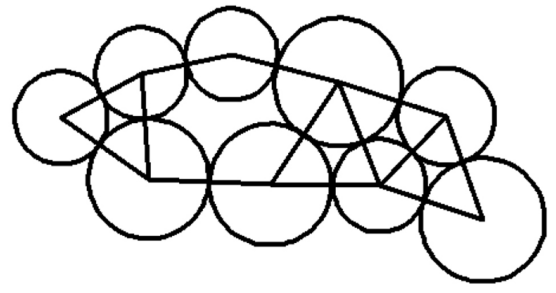

(b)

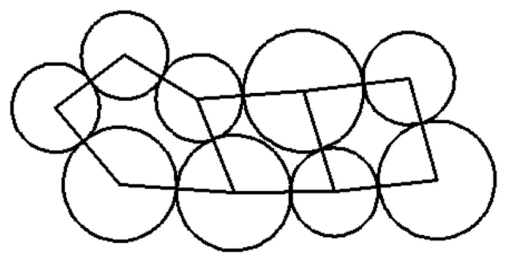

(c)

Figure 13. Evolution of structure of cycle. (a) The initial structure; (b) Structural failure; (c) The new stable structure.

structure of soil particles of slope is shown as Figure 13(b), where the particles of soil slope will rearrange, structures of 3-cycle are damaged and transformed into some parts of structures of 4-cycle and 5-cycle, and thus the number of 3 -cycle reduced accompanied by increasing number of 4-cycle and 5-cycle causes network to become more unstable and the shear strength of slope decreases sharply.

Especially, however, when $f=0.9$, the number of 4-cycle decreases with increasing time steps, the reason for which is that: there are less amounts of particles sliding in the process of landslide for the slope with $f$ of 0.9 , and its network structure of particles is shown in Figure 13(b); there small amounts of 3-cycle are damaged and combined with adjacent structures of 4-cycle to rearrange and form 5-cycle, which makes the numbers of 3-cycle and 4-cycle decrease and number of 5-cycle increase, and thus the number of 5-cycle at stable stage of landslide is the most. For the values of $f$ are $0.2,0.3$ and 0.5 , there are more particles sliding in the process of landslide, which cause more particles rearrange and form the network structure shown in Figure 13(c), so the number of 4-cycle increases sharply.

During the process of landslide, for the slopes with different values of $f$, with decreasing values of $f$, the number of 3-cycle decreases and those of 4-cycle and 
5-cycle increase at the same computational time steps. The reason for the phenomena is that: for the different values of $f$, the amounts of displacement of slope post-failure are different and the number of cycles damaged is different in the process of landslide. For a contact network, both stricture and number of cycle together determine the stability of network. When $f=0.2$, although the number of 3-cycle decreases, the numbers of 4-cycle and 5-cycle increase in the process of landslide. Compared with other cycle, however, the number of 3-cycle in the network is still the most, so its stability is controlled by the stability of 3-cycle. With the reduction of values of $f$, the displacement of soil slope increases, the number of 3-cycle decreases, and those of 4-cycle and 5-cycle increase, leading to the decreasing stability of soil slope.

\subsection{Analysis on Relationships Bridging Meso and Macro Parameters}

During the process of landslide, the relationships between meso parameters and macro parameters can be obtained based on the above analysis on the evolution of degree, clustering coefficient and $\mathrm{N}$-cycle. The evolution relationship between the average degree and displacement of slope toe is shown in Figure 14, from which we can find that during the process of landslide, with increasing $f$ the average degree decreases fast within small displacement of slope toe, and then when the displacement of slope toe becomes bigger, the average degree decreases. Therefore, monitoring the variation of the average degree of soil particles can predict the slide displacement of slope.

When the slope reaches a new stable state post-failure, the relationship between clustering coefficients and inclination of angle at stable state of slope can be obtained and shown in Figure 15. From Figure 15, we can find that with increasing $f$, both inclination angle and clustering coefficient increase at a new stable state. When $f$ is smaller than 0.3 , the variation of $\mathrm{f}$ affects greatly on clustering coefficient and inclination angle; however, when $f$ is more than 0.3 , the variation of $f$ affects slightly on clustering coefficient and inclination angle.

Figure 16 presents the evolution relationship between displacement of slope

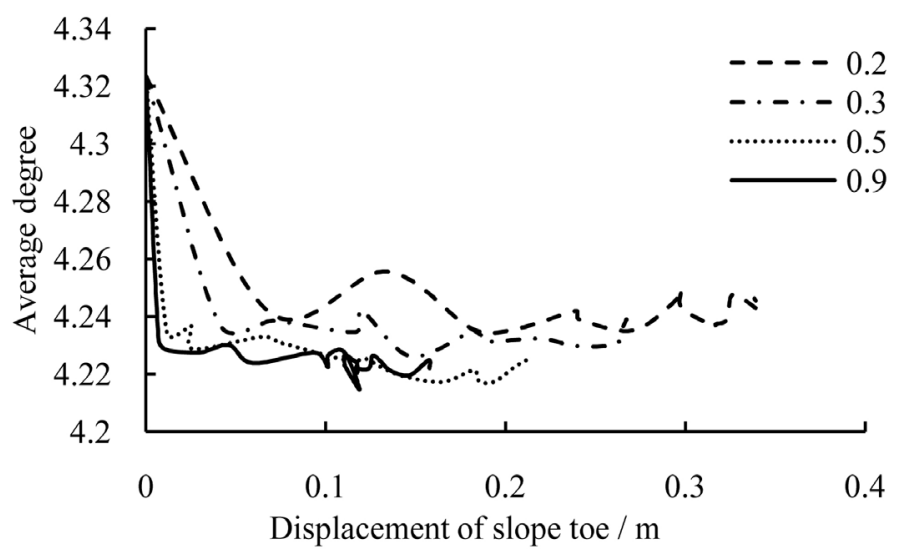

Figure 14. Evolution of average degree with displacement of slope toe. 


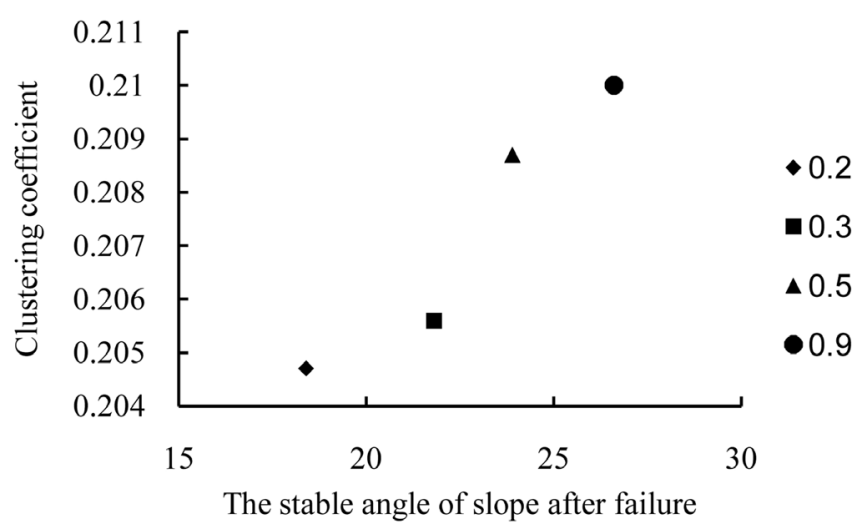

Figure 15. Relationship of clustering coefficient and the stable angle of slope after failure.

toe and N-cycle. From Figure 16(a), we can see that the number of 3-cycle decreases with increasing displacement of slope toe; within small displacement of slope toe, the number of 3-cycle reduces linearly for all the different $f$; with decreasing $f$, the number of 3-cycle reduces more sharply. When the displacement of slope toe is the same, the number of 3-cycle becomes larger with the decrease of $f$. From Figure 16(b) we can see that when $f$ is 0.2 or 0.3 , the number of 4 -cycle increases with the increasing displacement of slope toe; when $\mathrm{f}$ is 0.5 , the number of 4 -cycle changes slightly; and when $\mathrm{f}$ is 0.9 , the number of 4 -cycle decreases with increasing displacement of slope toe. For the same displacement of slope toe, the number of 4 -cycle decreases with increasing $f$. From Figure 16(c), the number of 5-cycle increases with increasing displacement of slope toe; when the displacement of slope toe is small, the number of 5-cycle increases linearly; with decreasing $f$, the increasing rate of 5-cycle becomes slow.

\section{Conclusions}

Based on discrete element method, the landslide processes of slope with four sets of inter-particle frictional coefficients are simulated and the evolution of meso structures is analyzed in the perspective of complex network. The conclusions can be summarized as follows.

1) The inter-particle coefficient has a great influence on the displacement of slope and evolutions of meso structure parameters, including the average degree, the clustering coefficient, and $\mathrm{N}$-cycle $(\mathrm{N}=3,4,5)$, during the sliding process.

2) With increasing inter-particle frictional coefficients, the displacement decreases and the inclination angle formed at stable state of slope increases, the average degree becomes smaller at initial stage of landslide and decreases when reaching a new stable state, the clustering coefficients decrease all the time.

3) For the same computational time step, with decreasing $f$, the number of 3-cycle decreases, and those of 4-cycle and 5-cycle increase, leading to the decreasing stability of soil slope.

In this study we explore the evolution of meso parameter during landslide 


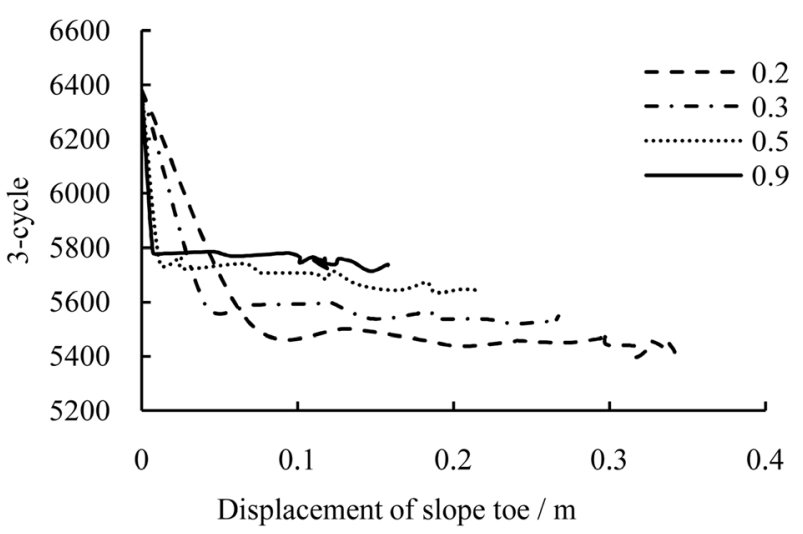

(a)

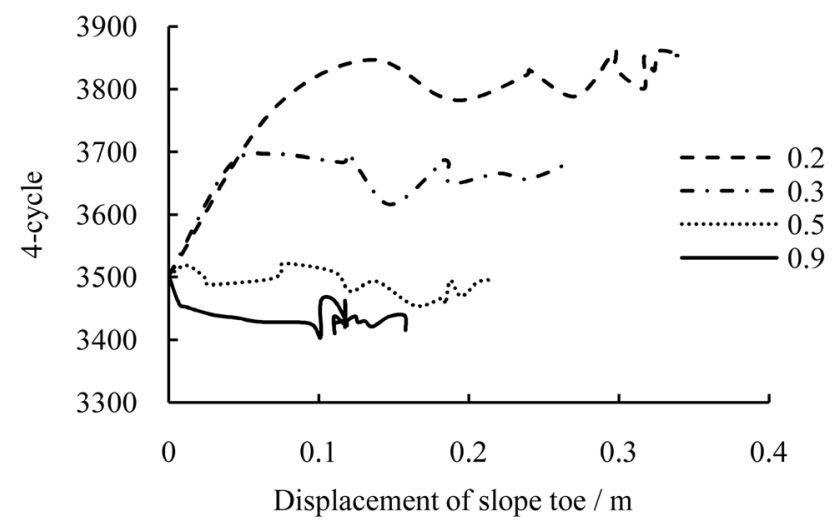

(b)

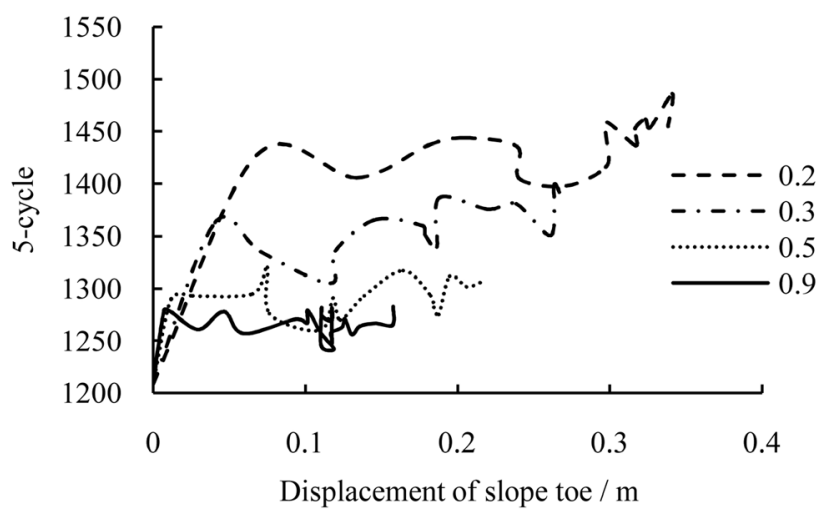

(c)

Figure 16. Evolution of N-cycle with displacement of slope toe. (a) 3-cycle; (b) 4-cycle; (c) 5-cycle.

process in a first step to establish the relationships bridging meso and macro parameters, such as evolution of average degree, clustering coefficient and evolution of N-cycle with displacement of slope toe. These meso parameters should be connected with the physical mechanism of landslide in further study to evaluate the evolution and movement of landslide.

\section{Compliance with Ethics Guidelines}

All authors declare that they have no conflict of interest or financial conflicts to 
disclose.

\section{References}

[1] Laouafa, F. and Darve, F. (2002) Modelling of Slope Failure by a Material Instability Mechanism. Computers \& Geotechnics, 29, 301-325. https://doi.org/10.1016/S0266-352X(01)00030-1

[2] Bishop, A.W. (1955) The Use of the Slip Circle in Stability Analysis of Slopes. Géotechnique, 5, 7-17. https://doi.org/10.1680/geot.1955.5.1.7

[3] Sarma, S.K. (1979) Stability Analysis of Embankments and Slopes. Journal of Geotechnical Engineering Division, ASCE, 105, 1511-1524.

[4] Clough, R.W. and Woodward, R.J. (1967) Analysis of Embankment Stresses and Deformations. Soil Mechanics \& Foundation Division, ASCE, 93, 529-549.

[5] Cundall, P.A. and Strack, O.D.L. (1979) A Discrete Numerical Mode for Granular Assemblies. Géotechnique, 29, 47-65. https://doi.org/10.1680/geot.1979.29.1.47

[6] Potyondy, D.O. and Cundall, P.A. (2004) A Bonded-Particle Model for Rock. International Journal of Rock Mechanics \& Mining Sciences, 41, 1329-1364. https://doi.org/10.1016/j.ijrmms.2004.09.011

[7] Li, X.P., He, S.M., Luo, Y. and Wu, Y. (2012) Simulation of the Sliding Process of Donghekou Landslide Triggered by the Wenchuan Earthquake Using a Distinct Element Method. Environmental Earth Sciences, 65, 1049-1054. https://doi.org/10.1007/s12665-011-0953-8

[8] Fu, Z.Z., Chen, S.S. and Liu, S.H. (2016) Discrete Element Simulations of Shallow Plate-Load Tests. International Journal of Geomechanics, 16, Article ID: 04015077. https://doi.org/10.1061/(ASCE)GM.1943-5622.0000588

[9] Camones Jr., L., E.D.A.V., Figueiredo, R.P.D. and Velloso, R.Q. (2013) Application of the Discrete Element Method for Modeling of Rock Crack Propagation and Coalescence in the Step-path Failure Mechanism. Engineering Geology, 153, 80-94. https://doi.org/10.1016/j.enggeo.2012.11.013

[10] Kun, F.,Varga, I., Lennartz-Sassinek, S. and Main, I.G. (2014) Rupture Cascades in a Discrete Element Model of a Porous Sedimentary Rock. Physical Review Letters, 112, Article ID: 065501. https://doi.org/10.1103/PhysRevLett.112.065501

[11] Wang, C., Tannant, D.D. and Lilly, P.A. (2003) Numerical Analysis of the Atability of Heavily Jointed Rock Slopes Using PFC2D. International Journal of Rock Mechanics \& Mining Sciences, 40, 415-424. https://doi.org/10.1016/S1365-1609(03)00004-2

[12] Scholtès, L. and Donzé, F.V. (2015) A DEM Analysis of Step-Path Failure in Jointed Rock Slopes. Comptes Rendus Mécanique, 343, 155-165. https://doi.org/10.1016/j.crme.2014.11.002

[13] Chen, H. and Liu, S.H. (2007) Slope Failure Characteristics and Stabilization Methods. Canadian Geotechnical Journal, 44, 377-391. https://doi.org/10.1139/t06-131

[14] Smith, D.A. and White, D.R. (1992) Structure and Dynamics of the Global Economy Network Analysis of International Trade 1965-1980. Social Forces, 70, 857-893. https://doi.org/10.1093/sf/70.4.857

[15] Doreian, P. and Mrvar, A. (2009) Partitioning Signed Social Networks. Social Networks, 31, 1-11. https://doi.org/10.1016/j.socnet.2008.08.001

[16] Andreasen, V. (2011) The Final Size of an Epidemic and Its Relation to the Basic Reproduction Number. Bulletin of Mathematical Biology, 73, 2305-2321. https://doi.org/10.1007/s11538-010-9623-3 
[17] Amaral, L. and Ottino, J.M. (2004) Complex Networks, Augmenting the Framework for the Study of Complex Systems. Physics of Condensed Matter, 38, 147-162.

[18] Peters, J.F., Muthuswamy, M., Wibowo, J. and Tordesillas, A. (2005) Characterization of Force Chains in Granular Material. Physical Review E, 72, Article ID: 041307. https://doi.org/10.1103/PhysRevE.72.041307

[19] Walker, D.M. and Tordesillas, A. (2010) Topological Evolution in Dense Granular Materials: A Complex Networks Perspective. International Journal of Solids \& Structures, 47, 624-639. https://doi.org/10.1016/j.ijsolstr.2009.10.025

[20] Tordesillas, A., O'Sullivan, P. and Walker, D.M. (2010) Evolution of Functional Connectivity in Contact and Force Chain Networks: Feature Vectors, K-Cores and Minimal Cycles. Comptes Rendus Mécanique, 338, 556-569. https://doi.org/10.1016/j.crme.2010.09.004

[21] Papadopoulos, L., Puckett, J.G., Daniels, K.E. and Bassett, D.S. (2016) Evolution of Network Architecture in a Granular Material under Compression. Physical Review E, 94, Article ID: 032908. https://doi.org/10.1103/PhysRevE.94.032908

[22] Jiang, M. and Murakami, A. (2012) Distinct Element Method Analyses of Idealized Bonded-Granulate Cut Slope. Granular Matter, 14, 393-410. https://doi.org/10.1007/s10035-012-0347-y

[23] Zhang, L.W. (2012) Influence of Anisotropic Internal Friction Angle on the Stability of Uniform Soil Slopes. Applied Mechanics \& Materials, 170-173, 270-273. https://doi.org/10.4028/www.scientific.net/AMM.170-173.270

[24] Utili, S. and Nova, R. (2008) DEM Analysis of Bonded Granular Geomaterials. International Journal for Numerical and Analytical Methods in Geomechanics, 32, 1997-2031. https://doi.org/10.1002/nag.728

[25] Viratjandr, C. and Michalowski, R.L. (2006) Limit Analysis of Submerged Slopes Subjected to Water Drawdown. Canadian Geotechnical Journal, 43, 802-814. https://doi.org/10.1139/t06-042

[26] Dorogovtsev, S.N. and Mendes, J.F.F. (2004) The Shortest Path to Complex Networks. Physics, 71, 47-53.

[27] Costa, L.D.F., Rodrigues, F.A., Travieso, G. and Boas, P.R.V. (2007) Characterization of Complex Networks: a Survey of Measurements. Advances in Physics, 56, 167-242. https://doi.org/10.1080/00018730601170527

[28] Bollobás, B. (1960) Modern Graph Theory. Springer, New York.

\section{Abbreviations}

\begin{tabular}{cc}
\hline DEM & discrete element method \\
PFC & particle flow code \\
$f$ & inter-particle frictional coefficient \\
$k$ & the average degree \\
C & the clustering coefficient \\
\hline
\end{tabular}

